\title{
壳-核型离子交换剂粒内扩散传质 理论计算及应用
}

\author{
马永利邱陵 岳廷盛
}

(兰州大学材料科学系)

\section{一、引亩}

和常规球状离子交换剂相比, 壳一核型交换剂能显著提高离子的传质速度,因而近 20 年来 得到了广泛的应用. 例如, 在高效色谱分离中薄壳型交换剂作为固定相便是其成功的一例. 不 过, 与传质速度得到提高相联系的是交换容量的必将降低. 对分析分离而言, 后一影响可以不 计, 但对制备分离及常量物料富集, 则希望传质速度和交换容量同时兼顾, 因此需适当选择这 类交换剂核所占的体积, 以适应不同场合的需要. 为从理论上计算出売一核型交换剂几何特征 参数对内扩散传质参量及交换容量的关系, 本文研究了壳-核型交换剂的内扩散传质动力学, 推导出不同几何特征参数下的 $F-B t$ 近似式,它不仅可用于求算内扩散常数 $B$ 和内扩散系数 $\bar{D}$, 而且可求算出交换剂的几何特征参数与半交换期和交换容量的关系, 为前者的选取提供理 论基础.

\section{二、内扩散传质交换度方程的近似式}

设球状壳一核型交换剂的球径为 $r_{0}$, 作为惰性载体的核径为 $r_{0}-d, d$ 是壳-交换体的沿 径厚度, $d \leqslant r_{0}$.

壳内扩散方程服从 Fick 第二定律:

$$
\frac{\partial \bar{c}}{\partial t}=\bar{D}\left(\frac{\partial^{2} \bar{c}}{\partial r^{2}}+\frac{2}{r} \cdot \frac{\partial \bar{c}}{\partial r}\right)
$$

式中 $\bar{c}$ 为某离子的粒内浓度.

当同位素离子作互扩散时, $\bar{D}$ 严格不依时间和地点而变, 称自扩散系数. 在不同离子互 扩散时, 只要扩散离子的浓度足够低(通常认为扩散离子所占的交换量低于交换剂总交换量的 $3 \%$ ), 使交换引起的粒内化学组成的改变对扩散电势、活度系数的影响可以忽略时, $\widetilde{D}$ 近似 为一常数 ${ }^{[1,2]}$.

今研究某离子的解吸过程. 边界条件如下: 由于无离子由核内进人壳内,所以

$$
\left.\frac{\partial \bar{c}}{\partial r}\right|_{r=r_{0}-d}=0 .
$$

令解吸剂高速通过交换剂, 将使液膜厚度减缩到液膜扩散过程可以忽略; 同时, 过程开始 后两相界面处的浓度趋于零, 所以

$$
\left.\bar{c}\right|_{r=r_{0}}=0
$$

本文 1986 年 9 月 27 日收到. 
起始条件为

$$
\left.\bar{c}\right|_{t=0}-\bar{c}^{0} \text {. }
$$

式中 $\check{c}^{0}$ 是解吸前离子的粒内浓度.

按上述边界及起始条件，可求得方程 (1) 的解，再对它在挬内体积分，进而得到

$$
F(t)=1-6 \cdot \frac{1-\lambda_{0}}{1+\lambda_{0}+\lambda_{0}^{2}} \sum_{n=1}^{\infty} \frac{1}{\mu_{n}^{2}} \cdot \frac{\exp \left(-\frac{\mu_{n}^{2}}{\pi^{2}} B t\right)}{1-\lambda_{0} \sin ^{2} \mu_{n}} .
$$

上述交换度理论方程已经前人 导出 ${ }^{[3]}$,

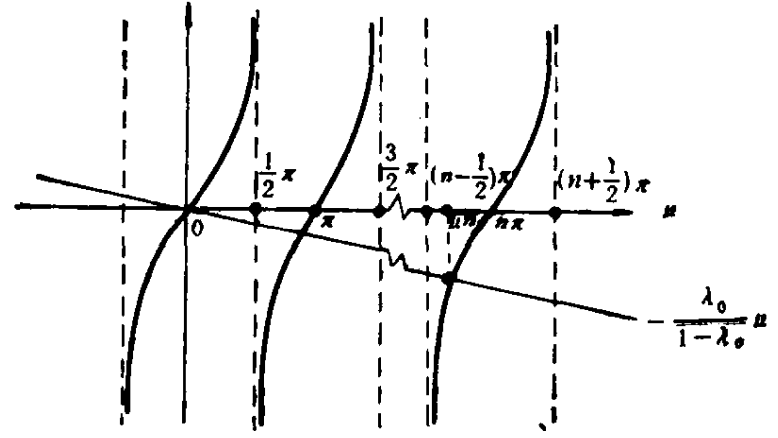

图， $\mu_{x}$ 的图解法 式中 $F(t)$ 是 $t$ 时刻的交换度, $B-\frac{\pi^{2}}{d^{2}} \bar{D}$, 称扩散常数; 核径对 $r_{0}$ 之比 $\lambda_{0}-1-\frac{d}{r_{0}}$, 称 为几何特征参数; 本征值 $\mu_{n}$ 是方程 $\mathrm{tg} \mu=$ $-\frac{\lambda_{0}}{1-\lambda_{0}} \mu$ 的非零正根 $(n-1,2,3, \cdots$. 参见图 1).

但方程 (5) 过于繁杂而不切实用, 故本 文研究了其近似分析解如下:

按 (5) 式, $F(t)$ 对 $B t$ 的偏导为

$$
\frac{\partial F(t)}{\partial(B t)}=\frac{6}{\pi^{2}} \cdot \frac{1-\lambda_{0}}{1+\lambda_{0}+\lambda_{0}^{2}} \cdot \sum_{n=1}^{-} \frac{\exp \left(-\frac{\mu_{n}^{2}}{\pi^{4}} B t\right)}{I-\lambda_{0} \sin ^{2} \mu_{n}} .
$$

先研究 $\lambda_{0}$ 足够小的情况, 此时的 $\operatorname{tg} \mu \approx 0$, 亦即 $\mu_{n} \approx n \pi ，(6)$ 式简化为

$$
\frac{\partial F(t)}{\partial(B t)} \approx \frac{6}{\pi^{2}} \cdot \frac{1-\lambda_{0}}{1+\lambda_{0}+\lambda_{0}^{2}} \cdot \frac{1}{2}\left(\sqrt{\frac{\pi}{B t}}-1\right) .
$$

把 (7) 式对 $B t$ 从 0 到 $B t$ 积分, 且 $F(0)=0$, 则有

$$
F(t)=\frac{6\left(1-\lambda_{0}\right)}{1+\lambda_{0}+\lambda_{0}^{2}} \sqrt{\frac{B t}{\pi^{3}}}-\frac{3\left(1-\lambda_{0}\right)}{1+\lambda_{0}+\lambda_{0}^{2}} \cdot \frac{B t}{\pi^{2}} .
$$

对于 $\lambda_{0}=0$, 即常规的离子交换球的情况,上式简化为

$$
B t-2 \pi-\frac{\pi^{2}}{3} F-2 \pi \sqrt{1-\frac{\pi}{3} F},
$$

(9) 式与 Reichenberg ${ }^{(4)}$ 利用 Poisson 公式所得的结果完全一致.

对 $\lambda_{0}=1$, 即相应于薄壳离子交换剂的另一极端情况, 此时 $\mu_{n} \approx\left(n-\frac{1}{2}\right) x$, 同理有

$$
B t=\left(1+\lambda_{0}+\lambda_{0}^{2}\right)^{2} \frac{\pi^{3}}{36} F^{2},
$$

与文献 [3] 的结果完全一致.

对于 $0<\lambda_{0}<1$ 的情况, 应先讨论 $\mu$ 值, 如图 1 所示. $\mu_{n}$ 位于 $\mu=\left(n-\frac{1}{2}\right) \pi$ 到 $n \pi$ 之 间, 当 $\lambda_{0} \geqslant 2 / 3$ 时, 其值收玫很快, 这时仅需取 $\mu_{1}$ 的精确解, 而 $\mu_{n}$ 取 $\left(n-\frac{1}{2}\right) \pi(n=2,3$, $4, \cdots)$, 则 


$$
\frac{\partial F(t)}{\partial(B t)}=\frac{6}{\pi^{2}} \cdot \frac{1-\lambda_{0}}{1+\lambda_{0}+\lambda_{0}^{2}}\left\{\frac{\exp \left(-\frac{\mu_{1}^{2}}{\pi^{2}} B t\right)}{1-\lambda_{0} \sin ^{2} \mu_{1}}+\frac{1}{1-\lambda_{0}}\left[\frac{1}{2} \sqrt{\frac{\pi}{B t}}-\exp \left(-\frac{1}{4} B t\right)\right]\right\} .
$$

积分后得到

$$
\begin{aligned}
F- & \frac{6}{\pi^{3 / 2}} \cdot \frac{\sqrt{B t}}{1+\lambda_{0}+\lambda_{0}^{2}}+6 \cdot \frac{1-\lambda_{0}}{1+\lambda_{0}+\lambda_{0}^{2}}\left\{\frac{1-\exp \left(-\frac{\mu_{1}^{2}}{\pi^{2}} \cdot B t\right)}{\mu_{1}^{2}\left(1-\lambda_{0} \sin ^{2} \mu_{1}\right)}\right. \\
& \left.-\frac{4}{1-\lambda_{0}}\left[1-\exp \left(-\frac{1}{4} B t\right)\right]\right\},
\end{aligned}
$$

当 $B t$ 足够小时, 上式简化为

$$
B_{t}=\frac{\pi^{2}}{4 g_{1}^{2}}\left[1-\sqrt{1-\frac{2 \pi}{3}\left(1+\lambda_{0}+\lambda_{0}^{2}\right) g_{1} F}\right]^{2},
$$

式中 $g_{1}-\lambda_{0} \cos ^{2} \mu_{1} /\left(1-\lambda_{0} \sin ^{2} \mu_{1}\right)$.

当 $\lambda_{0}<2 / 3$ 时, 需取前 $l$ 个 $\mu_{n}$ 的精确解, 其余的取 $\left(n-\frac{1}{2}\right) x(n=l+1, l+2, \cdots)$, 则

$$
\begin{aligned}
F- & \frac{6}{\pi^{3 / 2}} \cdot \frac{B t}{1+\lambda_{0}+\lambda_{0}^{2}}+\frac{6\left(1-\lambda_{0}\right)}{1+\lambda_{0}+\lambda_{0}^{2}} \\
& \times \sum_{n=1}^{l}\left\{\frac{1-\exp \left(-\frac{\mu_{n}^{2}}{\pi^{2}} B t\right)}{\mu_{n}^{2}\left(1-\lambda_{0} \sin ^{2} \mu_{n}\right)}-\frac{\left[1-\exp \left(-\frac{\mu_{0}^{2}}{\pi^{2}} B t\right)\right]}{\left(n-\frac{1}{2}\right)^{2}\left(1-\lambda_{0}\right)}\right\} .
\end{aligned}
$$

当 $B t$ 足够小时, 上式同样简化为 (13) 式, 但 $g_{1}=\sum_{n=1}^{l} \lambda_{0} \cos ^{2} \mu_{n} /\left(1-\lambda_{0} \sin ^{2} \mu_{n}\right)$.

在 $\lambda_{0}$ 从 0 到 1 的范围内, 若 $B t$ 足够大时, (5) 式仅取求和第 1 项,得

$$
B t=\frac{\pi^{2}}{\mu_{1}^{2}} \ln \left[\frac{6\left(1-\lambda_{0}\right)}{\left(1+\lambda_{0}+\lambda_{0}^{2}\right) \mu_{1}^{2}\left(1-\lambda_{0} \sin ^{2} \mu_{1}\right)} \cdot \frac{1}{1-F}\right] .
$$

(8) - (10)、(12)-(15) 式就是不同情况下近似的交换度理论方程.

\section{三、应用}

1. 壳-核型交换剂内扩散系数的测算: 以玻球为核、用微晶 $\mathrm{Ti}\left(\mathrm{HPO}_{4}\right)_{2} \cdot \frac{1}{2} \mathrm{H}_{2} \mathrm{O}$ 粘结为 球壳, 平均的 $r_{0}=150 \mu \mathrm{m}, d=60 \mu \mathrm{m}$, 水溶涨 $H$ 式交换容量为 $0.164 \pm 0.07 \mathrm{meq} / \mathrm{ml}$. 用浅床 法 ${ }^{[4]}$ 进行实验.

因 $\lambda_{\mathrm{t}}=0.60$, 相应的 $F(t)-B t$ 关系如图 2 中 IV 所示. 一组典型的实验数据与求算的 $\bar{D}$ 如表 1. 表中 $\overline{\mathrm{H}}^{+}-\mathrm{Li}^{+}\left(\mathrm{oAc}^{-}\right)$表示交换剂中的 $\mathrm{H}^{+}$和液相中醋酸锂所解离的 $\mathrm{Li}^{+}$间的交 换, $\overline{\mathrm{Li}^{+}}-\mathrm{H}^{+}\left(\mathrm{oAc}^{-}\right)$是其逆过程.

2. 壳一核型交换剂几何特征参数 $\lambda_{0}$ 之选取: 从交换度理论方程可见, 交换度 $F$ 与 $B t$ 的关 系取决于几何特征参数 $\lambda_{0}$ 值, 用微机对 (14) 式进行数值计算 (切割误差小于 $0.05 \%$ ), 得到不 同 $\lambda_{0}$ 时的 $F-B t$ 关系曲线,结果在图 2 示出. 
表 1 売一校型交换斺中 $\mathrm{H}^{+} 、 \mathrm{Li}^{+}$互交换 $\bar{D}$ 值的溯算 $\left(\lambda_{0}=0.6035 .0 \pm 0.5{ }^{\circ} \mathrm{C}\right)$

\begin{tabular}{|c|c|c|c|c|c|c|c|c|c|}
\hline 交块过程 & \multicolumn{8}{|c|}{$F-t$ 的实猃值 } & $\bar{D}\left(\mathrm{~cm}^{2} / \mathrm{s}\right)$ \\
\hline \multirow{2}{*}{$\overline{\mathrm{H}}^{+}-\mathrm{Li}^{+}\left(o \mathrm{AC} c^{-}\right)$} & $x(s)$ & 30 & 90 & 210 & 390 & 690 & 990 & 1290 & \multirow{2}{*}{$1.9 \times 10^{-1}$} \\
\hline & $F$ & 0.224 & 0.405 & 0.538 & 0.656 & 0.791 & 0.900 & 1.00 & \\
\hline \multirow{2}{*}{$\overline{\mathrm{Li}}^{+}-\mathrm{H}^{+}\left(o \mathrm{Ac}^{-}\right)$} & $t(s)$ & 30 & 90 & 210 & 330 & 510 & 690 & 870 & \multirow{2}{*}{$7.3 \times 10^{-8}$} \\
\hline & $F$ & 0.378 & 0.654 & 0.854 & 0.915 & 0.963 & 0.988 & 1.00 & \\
\hline
\end{tabular}

和 $F=0.5$ 相应的时间称为半交换期 $t_{1 / 2}$,

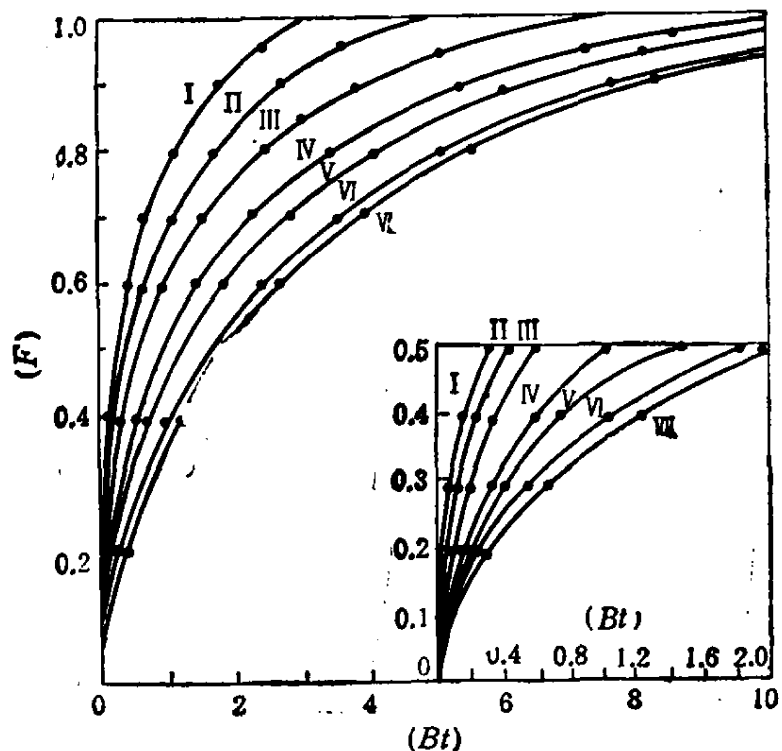

图 2 不同 $\lambda_{0}$ 的 $F-B t$ 关系曲线

$\lambda_{0}: 1.0$; II. 0.20 ; III. 0.40 ; IV. 0.60 ; V. 0.70; VI. 0.90 ; VII. 1.0

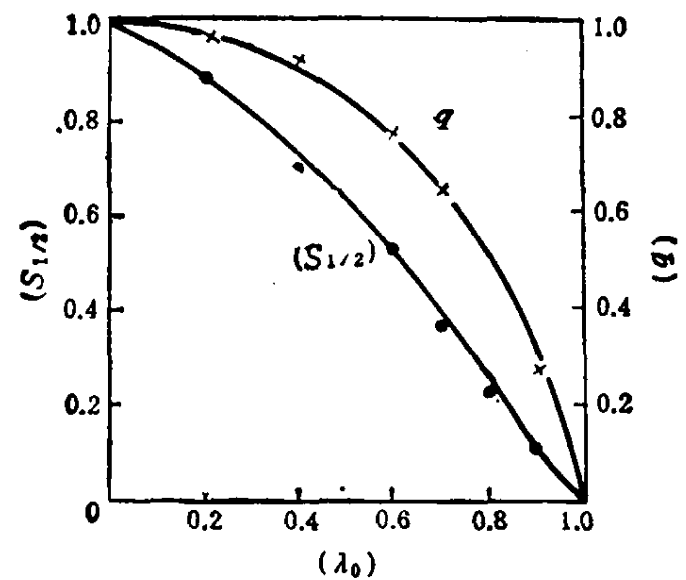

图 $3 q_{、} s_{1 / 2} \sim \lambda_{0}$ 的关系 它是一个宏观的有实用价值的传质参数, 它 决定于 $\lambda_{0}, r_{0}$ 和 $\bar{D}$. 试固定 $r_{0}$ 而改变 $d, t_{1 / n}$ 必随 $d$ 之减小（或 $\lambda_{0}$ 之增加）而缩减，可把 $\lambda_{0}=\lambda_{i}$ 和 $\lambda_{0}=0$ 时的 $\lambda_{1 / 2}$ 之比 $\left(s_{1 / 2}\right)_{\lambda_{i}}=$ $\frac{\left(1-\lambda_{i}\right)^{2}}{0.30}\left(B t_{1 / 2}\right)_{\alpha_{i}}$ 称为相对半交换期, 式 中 $\left(B t_{1 / 2}\right)_{z_{i}}$ 可从图 2 中找到. 又交换容量 $Q$ 亦依赖于 $\lambda_{\theta}$, 同样, 相对交换容量 $q=$ $(Q)_{\lambda_{i}} /(Q)_{0}=\left(1-\lambda_{i}\right)^{3}, \lambda_{4} \sim s_{1 / n}$ 和 $\lambda_{0} \sim q$ 的关系示于图 3; 图 4 是 $q \sim s_{1 / 2}$ 关系曲 线.

显然图 3 提供了选取 $\lambda_{0}$ 的理论根据，而 从图 4 可见到 $d q / d s_{1 \Omega}$ 随 $\lambda_{0}$ 的递减而变小， 它表示在低 $\lambda_{0}$ 区, 牺牲较少的交换量可以使 传质速度显著提高。

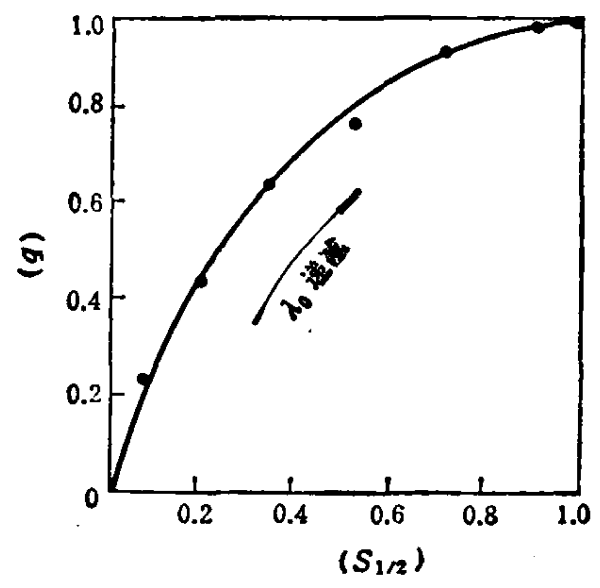

图 $4 \underset{s_{1 / 2}}{\text { 关系 }}$

\section{䒧文献}

[1] Helfferich, F., Ion Exchange, McGraw-Hill, New York, 1962, 266-283.

[ 2 ] Boyd, G. E., Adamson, A. W. and Myers, L. S., J. Amer. Chem. Soc, 69(1947), 2836-2848.

[ 3 ] Яскович Г. А., Кознева Е. Л., Елькин Г. Э., Воробьева В. Я., Сатсонов Г. В., Хим-Р.црл Жирн., 10(1974), 47-51.

[4] Reichenberg, D., J. Amer. Chem. Soc, 57(1953), 589. 